\title{
INCLUSION RESULTS FOR CONVOLUTION SUBMETHODS
}

\author{
JEFFREY A. OSIKIEWICZ and MOHAMMAD K. KHAN
}

Received 30 January 2003 and in revised form 9 June 2003

\begin{abstract}
If $B$ is a summability matrix, then the submethod $B_{\lambda}$ is the matrix obtained by deleting a set of rows from the matrix $B$. Comparisons between Euler-Knopp submethods and the Borel summability method are made. Also, an equivalence result for convolution submethods is established. This result will necessarily apply to the submethods of the Euler-Knopp, Taylor, Meyer-König, and Borel matrix summability methods.
\end{abstract}

2000 Mathematics Subject Classification: 40C05, 40D25, 40G05, 40G10.

1. Introduction and notation. Let $E$ be an infinite subset of $\mathbb{N} \cup\{0\}$ and consider $E$ as the range of a strictly increasing sequence of nonnegative integers, say $E:=\{\lambda(n)\}_{n=0}^{\infty}$. If $B:=\left(b_{n, k}\right)$ is a summability matrix, then the submethod $B_{\lambda}$ is the matrix whose $n k$ th entry is $B_{\lambda}[n, k]:=b_{\lambda(n), k}$. Thus, for a given sequence $x$, the $B_{\lambda}$-transform of $x$ is the sequence $B_{\lambda} x$ with

$$
\left(B_{\lambda} x\right)_{n}=(B x)_{\lambda(n)}:=\sum_{k=0}^{\infty} b_{\lambda(n), k} x_{k}
$$

Since $B_{\lambda}$ is a row submatrix of $B$, it is regular (i.e., limit preserving) whenever $B$ is regular.

Row submatrices have appeared throughout the literature [5, 6, 8, 12], but they were first studied as a class unto themselves by Goffman and Petersen [7], and later by Steele [14]. The class of Cesàro submethods has been studied by Armitage and Maddox [1] and Osikiewicz [11].

Let $A$ and $B$ be two summability matrices. If every sequence which is $A$-summable is also $B$-summable to the same limit, then $B$ includes $A$, denoted by $A \subseteq B$. Also, $B$ is called a triangle if $b_{n, k}=0$ for all $k>n$ and $b_{n, n} \neq 0$ for all $n$. The following lemma extends [1, Theorem 1].

LEMMA 1.1. Let $B$ be a summability matrix and let $E:=\{\lambda(n)\}$ and $F:=\{\rho(n)\}$ be infinite subsets of $\mathbb{N} \cup\{0\}$.

(1) If $F \backslash E$ is finite, then $B_{\lambda} \subseteq B_{\rho}$.

(2) If $B$ is a triangle and $B_{\lambda} \subseteq B_{\rho}$, then $F \backslash E$ is finite.

(3) If $B$ is a triangle, then $B_{\lambda}$ is equivalent to $B_{\rho}$ if and only if the symmetric difference $E \triangle F$ is finite.

In particular, $B \subseteq B_{\lambda}$ for any $\lambda$.

Proof. Assume $F \backslash E$ is finite and let $x$ be a sequence that is $B_{\lambda}$-summable to $L$. Then there exists an $N$ such that $\{\rho(n): n \geq N\} \subseteq E$. That is, $\{\rho(n): n \geq N\}$ is a 
subsequence of $\{\lambda(n)\}$. Since $\lim _{n}\left(B_{\lambda} x\right)_{n}=\lim _{n}(B x)_{\lambda(n)}=L$, we have $\lim _{n}\left(B_{\rho} x\right)_{n}=$ $\lim _{n}(B x)_{\rho(n)}=L$.

Now assume $B$ is a triangle, and hence invertible, and $F \backslash E$ is infinite. Let $F \backslash E:=$ $\{\rho(n(j))\}_{j=0}^{\infty}$ with $\rho(n(j))<\rho(n(j+1))$. Consider the sequence $y$ defined by

$$
y_{k}:= \begin{cases}(-1)^{j}, & \text { if } k=\rho(n(j)) \text { for some } j \\ 0, & \text { otherwise }\end{cases}
$$

and let $x$ be the sequence $B^{-1} y$. Then, for every $n$,

$$
\left(B_{\lambda} x\right)_{n}=(B x)_{\lambda(n)}=\left(B\left(B^{-1} y\right)\right)_{\lambda(n)}=y_{\lambda(n)}=0
$$

Hence, $\lim _{n}\left(B_{\lambda} x\right)_{n}=0$. However, for every $j$,

$$
\left(B_{\rho} x\right)_{n(j)}=(B x)_{\rho(n(j))}=\left(B\left(B^{-1} y\right)\right)_{\rho(n(j))}=y_{\rho(n(j))}=(-1)^{j} .
$$

Thus $x$ is not $B_{\rho}$-summable. Therefore $B_{\rho}$ does not include $B_{\lambda}$, which completes the contrapositive of assertion (2). Lastly, assertion (3) follows from (1) and (2) since $E \triangle F:=$ $(E \backslash F) \cup(F \backslash E)$.

To show the reason for the necessity of $B$ being a triangle in assertion (2) of Lemma 1.1 , consider the matrix $B$ whose $n k$ th entry is

$$
B[n, k]:= \begin{cases}0, & \text { if } n \text { even and } k \neq \frac{n}{2}, \\ 1, & \text { if } n \text { even and } k=\frac{n}{2} \\ 0, & \text { if } n \text { odd and } n \neq k \\ 1, & \text { if } n \text { odd and } n=k .\end{cases}
$$

Then if $\lambda(n):=2 n$ and $\rho(n):=2 n+1, F \backslash E$ is infinite and $B_{\lambda} \subseteq B_{\rho}$.

2. Inclusion results for Euler-Knopp submethods. For $r \in \mathbb{C} \backslash\{0,1\}$, the EulerKnopp method of order $r$ is given by the matrix $E_{r}$ whose $n k$ th entry is

$$
E_{r}[n, k]:= \begin{cases}\left(\begin{array}{l}
n \\
k
\end{array}\right) r^{k}(1-r)^{n-k}, & \text { if } k \leq n, \\
0, & \text { if } k>n .\end{cases}
$$

For the case $r=1, E_{1}$ is the identity matrix, and $E_{0}$ is the matrix whose $n k$ th entry is

$$
E_{0}[n, k]:= \begin{cases}1, & \text { if } k=0, n=0,1,2, \ldots, \\ 0, & \text { otherwise. }\end{cases}
$$

It is well known that $E_{r}$ is regular if and only if $0<r \leq 1$ (see [4]). 
Let $E:=\{\lambda(n)\}$ be an infinite subset of $\mathbb{N} \cup\{0\}$ and $r \in \mathbb{C} \backslash\{0,1\}$. The submethod $E_{r, \lambda}$ is the matrix whose $n k$ th entry is

$$
E_{r, \lambda}[n, k]:= \begin{cases}\left(\begin{array}{c}
\lambda(n) \\
k
\end{array}\right) r^{k}(1-r)^{\lambda(n)-k}, & \text { if } k \leq \lambda(n), \\
0, & \text { if } k>\lambda(n) .\end{cases}
$$

Then $E_{r, \lambda}$ is regular if and only if $E_{r}$ is regular.

By a direct application of Lemma 1.1, we have the following inclusion result for the $E_{r, \lambda}$ methods.

LEMMA 2.1. Let $E:=\{\lambda(n)\}$ and $F:=\{\rho(n)\}$ be infinite subsets of $\mathbb{N} \cup\{0\}$ and $r \neq 0$.

(1) The method $E_{r, \lambda} \subseteq E_{r, \rho}$ if and only if $F \backslash E$ is finite.

(2) The method $E_{r, \lambda}$ is equivalent to $E_{r, \rho}$ if and only if the symmetric difference $E \triangle F$ is finite.

We now examine the relationship between $E_{r, \lambda}$ and the Borel summability method. Recall that a sequence $x$ is Borel summable to $L$ if

$$
\lim _{t \rightarrow \infty} e^{-t} \sum_{k=0}^{\infty} x_{k} \frac{t^{k}}{k !}=L
$$

THEOREM 2.2. Let $E:=\{\lambda(n)\}$ be an infinite subset of $\mathbb{N} \cup\{0\}$ and $r>0$. Then the Borel summability method includes $E_{r, \lambda}$ if and only if $S:=(\mathbb{N} \cup\{0\}) \backslash E$ is finite.

Proof. If $S$ is finite, then by Lemma 2.1, $E_{r}$ and $E_{r, \lambda}$ are equivalent. But the Borel summability method includes $E_{r}$ for $r>0$ (see [4]). Hence, it also includes $E_{r, \lambda}$. If $S$ is infinite, then it may be written as a strictly increasing sequence of nonnegative integers, say $S:=\{\rho(m)\}_{m=0}^{\infty}$. If $M_{n}:=\max _{0 \leq k \leq n}\left|E_{r}[n, k]\right|$, consider the sequence $y$ defined by

$$
y_{n}:= \begin{cases}(\rho(m) !)^{2}(\rho(m)+1) M_{\rho(m)}, & \text { if } n=\rho(m), \\ 0, & \text { otherwise, }\end{cases}
$$

and let $x$ be the sequence $E_{r}^{-1} y$; that is, $y=E_{r} x$ and

$$
\lim _{n \rightarrow \infty}\left(E_{r, \lambda} x\right)_{n}=\lim _{n \rightarrow \infty}\left(E_{r} x\right)_{\lambda(n)}=\lim _{n \rightarrow \infty} y_{\lambda(n)}=0 .
$$

Hence, $x$ is $E_{r, \lambda}$-summable to 0 . Now observe that for a given $n$,

$$
\left|y_{n}\right|=\left|\left(E_{r} x\right)_{n}\right| \leq \sum_{k=0}^{n}\left|E_{r}[n, k]\right|\left|x_{k}\right| \leq M_{n} \sum_{k=0}^{n}\left|x_{k}\right|
$$


Thus, for $n=\rho(m)$, we have

$$
\begin{aligned}
(\rho(m) !)^{1 / \rho(m)} & =\left(\frac{1}{\rho(m) !} \cdot \frac{1}{\rho(m)+1} \cdot \frac{\left|y_{\rho(m)}\right|}{M_{\rho(m)}}\right)^{1 / \rho(m)} \\
& \leq\left(\frac{1}{\rho(m) !} \cdot \frac{1}{\rho(m)+1} \sum_{k=0}^{\rho(m)}\left|x_{k}\right|\right)^{1 / \rho(m)} .
\end{aligned}
$$

Since $\lim \sup _{m}(\rho(m) !)^{1 / \rho(m)}=\infty$,

$$
\limsup _{m \rightarrow \infty}\left(\frac{1}{\rho(m) !} \cdot \frac{1}{\rho(m)+1} \sum_{k=0}^{\rho(m)}\left|x_{k}\right|\right)^{1 / \rho(m)}=\infty
$$

and it follows that $\lim \sup _{n}\left(\left|x_{n}\right| / n !\right)^{1 / n}=\infty$. Thus, $\sum_{k=0}^{\infty}\left(x_{k} / k !\right) t^{k}$ diverges for all nonzero $t$ and hence $x$ is not Borel summable.

THEOREM 2.3. There exists a sequence which is Borel summable but not $E_{r, \lambda}$-summable for any $\lambda$ and $r>0$.

Proof. Let $r>0$ and consider the sequence $x$ defined by

$$
x_{n}:=n\left(-\frac{1}{r}\right)\left(1-\frac{2}{r}\right)^{n-1}
$$

Then it can be shown that $\left(E_{r, \lambda} x\right)_{n}=(-1)^{\lambda(n)} \lambda(n)$. Hence $x$ is not $E_{r, \lambda}$-summable for any $\lambda$. However,

$$
\begin{aligned}
e^{-t} \sum_{k=0}^{\infty} x_{k} \frac{t^{k}}{k !} & =e^{-t} \sum_{k=1}^{\infty}\left[k\left(-\frac{1}{r}\right)\left(1-\frac{2}{r}\right)^{k-1}\right] \frac{t^{k}}{k !} \\
& =\left(-\frac{1}{r}\right) e^{-t} \sum_{k=1}^{\infty}\left(1-\frac{2}{r}\right)^{k-1} \frac{t^{k}}{(k-1) !} \\
& =\left(-\frac{1}{r}\right) t e^{-t} \sum_{k=0}^{\infty}\left(1-\frac{2}{r}\right)^{k} \frac{t^{k}}{k !} \\
& =\left(-\frac{1}{r}\right) t e^{-t} e^{(1-2 / r) t} \\
& =\left(-\frac{1}{r}\right) t e^{-(2 / r) t} .
\end{aligned}
$$

Since $r>0$,

$$
\lim _{t \rightarrow \infty} e^{-t} \sum_{k=0}^{\infty} x_{k} \frac{t^{k}}{k !}=\lim _{t \rightarrow \infty}\left(-\frac{1}{r}\right) t e^{-(2 / r) t}=0,
$$

and hence $x$ is Borel summable to 0 . 
3. Convolution methods. Let $p$ and $q$ be sequences of real numbers with $p_{k} \geq 0$, $q_{k} \geq 0, \sum_{k=0}^{\infty} p_{k}=1$, and $\sum_{k=0}^{\infty} q_{k}=1$. The convolution summability method is given by the matrix $C^{*}:=\left(c_{n, k}\right)$ whose $n k$ th entry is

$$
c_{n, k}:= \begin{cases}q_{k}, & \text { if } n=0, \\ \sum_{j=0}^{k} c_{n-1, j} p_{k-j}, & \text { if } n \geq 1 .\end{cases}
$$

It is clear that $C^{*}$ is a nonnegative matrix such that for every $n, \sum_{k=0}^{\infty} c_{n, k}=1$. Some classical summability matrices are examples of the matrix $C^{*}$. If $0 \leq r \leq 1, p:=\{1-$ $r, r, 0,0, \ldots\}$, and $q:=\{1,0,0, \ldots\}$, then $C^{*}$ is the Euler-Knopp method of order $r$. If $0 \leq r<1, p:=\left\{0,(1-r),(1-r) r,(1-r) r^{2}, \ldots\right\}$, and $q:=\left\{(1-r),(1-r) r,(1-r) r^{2}, \ldots\right\}$, then $C^{*}$ is the Taylor method of order $r$, denoted by $T_{r}$. If $0<r<1$ and $p:=q:=$ $\left\{(1-r),(1-r) r,(1-r) r^{2}, \ldots\right\}$, then $C^{*}$ is the Meyer-König method of order $r$, denoted by $S_{r}$. If $p:=q:=\{1 / k ! e\}$, then $C^{*}$ is the Borel matrix method $B^{*}$. Similar forms of the convolution method are known by different names, such as the random-walk method and Sonnenschein method. (Further information on all of these methods may be found in $[3,4,13]$.)

If $C^{*}$ is the convolution method formed from the sequences $p$ and $q$, then let

$$
\mu:=\sum_{j=0}^{\infty} j p_{j}, \quad v:=\sum_{j=0}^{\infty} j q_{j} .
$$

We note here that for the remainder of this work, $p$ and $q$ are nonnegative sequences whose sums are 1 , and $\mu$ and $v$ represent the sums in (3.2). Also, $c_{n, k}:=0$ whenever $k<0$.

We next present some preliminary results concerning the convolution method.

LEMMA 3.1. The convolution method $C^{*}$ is regular if and only if $p_{0}<1$.

Proof. See [9].

LEMMA 3.2. If $\mu<\infty$ and $\nu<\infty$, then for every $n$,

$$
\sum_{k=0}^{\infty} k c_{n, k}=n \mu+\nu .
$$

Proof. Note that for $n=0$, the result holds. So assume the result holds for some integer $n>0$. Then

$$
\begin{aligned}
\sum_{k=0}^{\infty} k c_{n+1, k} & =\sum_{k=0}^{\infty} k\left(\sum_{j=0}^{k} c_{n, j} p_{k-j}\right)=\sum_{j=0}^{\infty} c_{n, j} \sum_{k=j}^{\infty} k p_{k-j} \\
& =\sum_{j=0}^{\infty} c_{n, j}\left(\sum_{i=0}^{\infty} i p_{i}+j \sum_{i=0}^{\infty} p_{i}\right)=\sum_{j=0}^{\infty} \mu c_{n, j}+\sum_{j=0}^{\infty} j c_{n, j}=(n+1) \mu+v .
\end{aligned}
$$

By induction, the result follows. 
LEMMA 3.3. Let $C^{*}$ be the convolution method formed from the sequences $p$ and $q$ and $D^{*}:=\left(d_{n, k}\right)$ the convolution method formed from the sequences $p$ and $\tilde{q}:=\{1,0,0, \ldots\}$. Then for nonnegative integers $n, k$, and $j$,

$$
c_{n+j, k}=\sum_{i=0}^{k} c_{n, k-i} d_{j, i} .
$$

The proof of this lemma is a straightforward induction argument left to the reader.

LEMMA 3.4. Let $C^{*}$ be the convolution method formed from the sequences $p$ and $q$. If $\mu<\infty, v<\infty, 0<\sum_{j=0}^{\infty}(j-\mu)^{2} p_{j}$, and $\sum_{j=0}^{\infty} j^{3} p_{j}<\infty$, then

$$
\sum_{k=0}^{\infty}\left|c_{n, k+1}-c_{n, k}\right|=O\left(\frac{1}{\sqrt{n}}\right) .
$$

Proof. Let $D^{*}:=\left(d_{n, k}\right)$ be the convolution method formed from the sequences $p$ and $\tilde{q}:=\{1,0,0, \ldots\}$. We first prove that the result holds for $D^{*}$.

Let $\phi(t):=\left(\sqrt{2 \pi} e^{t^{2} / 2}\right)^{-1}$ and $x_{n, k}:=(k-n \mu) / \sigma \sqrt{n}$, where $\sigma^{2}:=\sum_{j=0}^{\infty}(j-\mu)^{2} p_{j}$. Then

$$
\begin{aligned}
\sqrt{n} \sum_{k=0}^{\infty}\left|d_{n, k+1}-d_{n, k}\right| \leq & \sqrt{n} \sum_{k=0}^{\infty}\left|d_{n, k+1}-\frac{1}{\sigma \sqrt{n}} \phi\left(x_{n, k+1}\right)\right| \\
& +\sqrt{n} \sum_{k=0}^{\infty}\left|\frac{1}{\sigma \sqrt{n}} \phi\left(x_{n, k+1}\right)-\frac{1}{\sigma \sqrt{n}} \phi\left(x_{n, k}\right)\right| \\
& +\sqrt{n} \sum_{k=0}^{\infty}\left|\frac{1}{\sigma \sqrt{n}} \phi\left(x_{n, k}\right)-d_{n, k}\right| .
\end{aligned}
$$

The first and the third terms on the right-hand side of the inequality are bounded by a result of Bikjalis and Jasjunas [2]. For the middle term, the mean value theorem yields

$$
\begin{aligned}
\sqrt{n} \sum_{k=0}^{\infty}\left|\frac{1}{\sigma \sqrt{n}} \phi\left(x_{n, k+1}\right)-\frac{1}{\sigma \sqrt{n}} \phi\left(x_{n, k}\right)\right| & =\frac{1}{\sigma} \sum_{k=0}^{\infty}\left|\phi^{\prime}\left(\xi_{n, k}\right)\right|\left(x_{n, k+1}-x_{n, k}\right) \\
& <\frac{K}{\sigma} \int_{\mathbb{R}}\left|\phi^{\prime}(t)\right| d t<\infty,
\end{aligned}
$$

where $\xi_{n, k} \in\left(x_{n, k}, x_{n, k+1}\right)$ and $K>0$ is some constant. Thus, the result holds for the convolution method $D^{*}$. Then, by Lemma 3.3,

$$
\begin{aligned}
\sum_{k=0}^{\infty}\left|c_{n, k+1}-c_{n, k}\right| & =\sum_{k=0}^{\infty}\left|\sum_{i=0}^{k+1} q_{k+1-i} d_{n, i}-\sum_{i=0}^{k} q_{k-i} d_{n, i}\right| \\
& =\sum_{k=0}^{\infty}\left|q_{k+1} d_{n, 0}+\sum_{i=1}^{k+1} q_{k+1-i} d_{n, i}-\sum_{i=0}^{k} q_{k-i} d_{n, i}\right|
\end{aligned}
$$




$$
\begin{aligned}
& \leq p_{0}^{n} \sum_{k=0}^{\infty} q_{k+1}+\sum_{k=0}^{\infty} \sum_{i=0}^{k} q_{k-i}\left|d_{n, i+1}-d_{n, i}\right| \\
& \leq p_{0}^{n}+\sum_{i=0}^{\infty}\left|d_{n, i+1}-d_{n, i}\right| \sum_{k=i}^{\infty} q_{k-i} \\
& =p_{0}^{n}+\sum_{i=0}^{\infty}\left|d_{n, i+1}-d_{n, i}\right|=O\left(\frac{1}{\sqrt{n}}\right) .
\end{aligned}
$$

4. Equivalence results for convolution submethods. Let $E:=\{\lambda(n)\}$ be an infinite subset of $\mathbb{N} \cup\{0\}$. The convolution submethod $C_{\lambda}^{*}$ is the matrix whose $n k$ th entry is

$$
C_{\lambda}^{*}[n, k]:=C^{*}[\lambda(n), k]
$$

LEMMA 4.1. The convolution submethod $C_{\lambda}^{*}$ is regular if and only if $p_{0}<1$.

Proof. If $p_{0}<1$, then $C^{*}$ is regular and hence $C_{\lambda}^{*}$ is also regular. Conversely, if $C_{\lambda}^{*}$ is regular and $p_{0}=1$, then $C_{\lambda}^{*}[n, k]=q_{k}$ for all $n$ and $k$. Since $\sum_{k=0}^{\infty} q_{k}=1$, there exists a $\hat{k}$ such that $q_{\hat{k}} \neq 0$. Then $\lim _{n} C_{\lambda}^{*}[n, \hat{k}]=q_{\hat{k}} \neq 0$, which contradicts the regularity of $C_{\lambda}^{*}$.

The following theorem compares $C_{\lambda}^{*}$ with $C^{*}$ for bounded sequences.

THEOREM 4.2. Let $C^{*}$ be the convolution method formed from the sequences $p$ and $q$ with $\mu<\infty, \nu<\infty, 0<\sum_{j=0}^{\infty}(j-\mu)^{2} p_{j}$, and $\sum_{j=0}^{\infty} j^{3} p_{j}<\infty$. Let $E:=\{\lambda(n)\}$ be an infinite subset of $\mathbb{N} \cup\{0\}$. If

$$
\lim _{n \rightarrow \infty} \frac{\lambda(n+1)-\lambda(n)}{\sqrt{\lambda(n)}}=0
$$

then $C^{*}$ and $C_{\lambda}^{*}$ are equivalent for bounded sequences.

Proof. By Lemma 1.1, $C^{*} \subseteq C_{\lambda}^{*}$ for any $\lambda$. So assume $\lim _{n}(\lambda(n+1)-\lambda(n)) / \sqrt{\lambda(n)}=$ 0 and let $x$ be a bounded sequence that is $C_{\lambda}^{*}$-summable to $L$. Consider the set $S:=$ $\{\rho(n)\}:=(\mathbb{N} \cup\{0\}) \backslash E$. If $S$ is finite, then Lemma 1.1 shows that $C_{\lambda}^{*}$ and $C^{*}$ are equivalent for all sequences. So assume $S$ is infinite. Then there exists an $N$ such that for $n \geq N$, $\rho(n)>\lambda(0)$. Since $E$ and $S$ are disjoint, for $n \geq N$, there exists an integer $m$ such that $\lambda(m)<\rho(n)<\lambda(m+1)$. We write $\rho(n):=\lambda(m)+j$, where $0<j<\lambda(m+1)-\lambda(m)$. Then, for $n \geq N$,

$$
\begin{aligned}
\left|\left(C_{\rho}^{*} x\right)_{n}-\left(C_{\lambda}^{*} x\right)_{m}\right| & =\left|\sum_{k=0}^{\infty} c_{\rho(n), k} x_{k}-\sum_{k=0}^{\infty} c_{\lambda(m), k} x_{k}\right| \\
& =\left|\sum_{k=0}^{\infty} c_{\lambda(m)+j, k} x_{k}-\sum_{k=0}^{\infty} c_{\lambda(m), k} x_{k}\right|
\end{aligned}
$$


By Lemma 3.3, this becomes

$$
\begin{aligned}
\left|\left(C_{\rho}^{*} x\right)_{n}-\left(C_{\lambda}^{*} x\right)_{m}\right| & =\left|\sum_{k=0}^{\infty}\left(\sum_{i=0}^{\infty} c_{\lambda(m), k-i} d_{j, i}\right) x_{k}-\sum_{k=0}^{\infty} c_{\lambda(m), k} x_{k}\right| \\
& =\left|\sum_{k=0}^{\infty} x_{k}\left[\left(\sum_{i=0}^{\infty} c_{\lambda(m), k-i} d_{j, i}\right)-\left(\sum_{i=0}^{\infty} c_{\lambda(m), k} d_{j, i}\right)\right]\right| \\
& \leq\|x\|_{\infty} \sum_{k=0}^{\infty} \sum_{i=0}^{\infty} d_{j, i}\left|c_{\lambda(m), k-i}-c_{\lambda(m), k}\right| \\
& =\|x\|_{\infty} \sum_{i=0}^{\infty} d_{j, i} \sum_{k=0}^{\infty}\left|\sum_{l=0}^{i-1} c_{\lambda(m), k-l}-c_{\lambda(m), k-l-1}\right| \\
& \leq\|x\|_{\infty} \sum_{i=0}^{\infty} d_{j, i} \sum_{k=0}^{\infty} \sum_{l=0}^{i-1}\left|c_{\lambda(m), k-l}-c_{\lambda(m), k-l-1}\right| \\
& =\frac{\|x\|_{\infty}}{\sqrt{\lambda(m)}} \sum_{i=0}^{\infty} d_{j, i} \sum_{l=0}^{i-1} \sqrt{\lambda(m)} \sum_{k=0}^{\infty}\left|c_{\lambda(m), k-l}-c_{\lambda(m), k-l-1}\right|
\end{aligned}
$$

By Lemma 3.4, there exists an $M>0$ such that

$$
\sqrt{\lambda(m)} \sum_{k=0}^{\infty}\left|c_{\lambda(m), k-l}-c_{\lambda(m), k-l-1}\right|<M
$$

Then, by Lemma 3.2,

$$
\left|\left(C_{\rho}^{*} x\right)_{n}-\left(C_{\lambda}^{*} x\right)_{m}\right| \leq \frac{\|x\|_{\infty}}{\sqrt{\lambda(m)}} \sum_{i=0}^{\infty} d_{j, i} \sum_{l=0}^{i-1} M=\frac{\|x\|_{\infty} M}{\sqrt{\lambda(m)}} \sum_{i=0}^{\infty} i d_{j, i} \leq \frac{\|x\|_{\infty} M}{\sqrt{\lambda(m)}} \cdot j \mu .
$$

Since $0<j<\lambda(m+1)-\lambda(m)$,

$$
\left|\left(C_{\rho}^{*} x\right)_{n}-\left(C_{\lambda}^{*} x\right)_{m}\right|<\|x\|_{\infty} M \mu \cdot \frac{\lambda(m+1)-\lambda(m)}{\sqrt{\lambda(m)}}=o(1) .
$$

Thus,

$$
0 \leq\left|\left(C_{\rho}^{*} x\right)_{n}-L\right| \leq\left|\left(C_{\rho}^{*} x\right)_{n}-\left(C_{\lambda}^{*} x\right)_{m}\right|+\left|\left(C_{\lambda}^{*} x\right)_{m}-L\right|=o(1)+o(1)=o(1) .
$$

Therefore, the sequence $C^{*} x$ may be partitioned into two disjoint subsequences, namely $\left(C_{\lambda}^{*} x\right)_{n}=\left(C^{*} x\right)_{\lambda(n)}$ and $\left(C_{\rho}^{*} x\right)_{n}=\left(C^{*} x\right)_{\rho(n)}$, each having the common limit $L$. Thus, $x$ must be $C^{*}$-summable to $L$, and hence $C^{*}$ and $C_{\lambda}^{*}$ are equivalent for bounded sequences.

The following theorem is a well-known result due to Meyer-König (see [10, Theorem 25]).

THEOREM 4.3. The methods $E_{r}(0<r<1), S_{r}(0<r<1), T_{r}(0<r<1)$, and the Borel method are equivalent for bounded sequences. 
Since the Euler-Knopp methods of order $0<r<1$, Taylor methods of order $0<$ $r<1$, Meyer-König methods of order $0<r<1$, and the Borel matrix method all have generating sequences satisfying the conditions in Theorem 4.2, the following corollary is immediate.

Corollary 4.4. Let $E:=\{\lambda(n)\}$ be an infinite subset of $\mathbb{N} \cup\{0\}$ and $0<r<1$. If $\lambda$ satisfies condition (4.6), then $E_{r, \lambda}, E_{r}, T_{r, \lambda}, T_{r}, S_{r, \lambda}, S_{r}, B_{\lambda}^{*}, B^{*}$, and the Borel method are all equivalent for bounded sequences.

The next theorem presents an equivalence relationship between the $C_{\lambda}^{*}$ submethods.

THEOREM 4.5. Let $C^{*}$ be the convolution method formed from the sequences $p$ and $q$ with $\mu<\infty, v<\infty, 0<\sum_{j=0}^{\infty}(j-\mu)^{2} p_{j}$, and $\sum_{j=0}^{\infty} j^{3} p_{j}<\infty$. Let $E:=\{\lambda(n)\}$ and $F:=\{\rho(n)\}$ be infinite subsets of $\mathbb{N} \cup\{0\}$. If

$$
\lim _{n \rightarrow \infty} \frac{\rho(n)-\lambda(n)}{\sqrt{\lambda(n)}}=0
$$

then $C_{\lambda}^{*}$ and $C_{\rho}^{*}$ are equivalent for bounded sequences.

Proof. Let $x$ be a bounded sequence and consider the sequences $M(n):=\max \{\lambda(n)$, $\rho(n)\}$ and $m(n):=\min \{\lambda(n), \rho(n)\}$. We write $M(n):=m(n)+j$, where $j:=M(n)-$ $m(n)$. For $n \geq 1$, we have

$$
\begin{aligned}
\left|\left(C_{\rho}^{*} x\right)_{n}-\left(C_{\lambda}^{*} x\right)_{n}\right| & =\left|\sum_{k=0}^{\infty} c_{\rho(n), k} x_{k}-\sum_{k=0}^{\infty} c_{\lambda(n), k} x_{k}\right| \\
& =\left|\sum_{k=0}^{\infty} c_{M(n), k} x_{k}-\sum_{k=0}^{\infty} c_{m(n), k} x_{k}\right| \\
& =\left|\sum_{k=0}^{\infty} c_{m(n)+j, k} x_{k}-\sum_{k=0}^{\infty} c_{m(n), k} x_{k}\right| .
\end{aligned}
$$

Then, as in the proof of Theorem 4.2, we have

$$
\begin{aligned}
\left|\left(C_{\rho}^{*} x\right)_{n}-\left(C_{\lambda}^{*} x\right)_{n}\right| & \leq O(1) \frac{j}{\sqrt{m(n)}}=O(1) \frac{M(n)-m(n)}{\sqrt{m(n)}} \\
& =O(1) \sqrt{\frac{\lambda(n)}{m(n)}} \frac{|\rho(n)-\lambda(n)|}{\sqrt{\lambda(n)}} \\
& =O(1) \cdot O(1) \cdot o(1)=o(1) .
\end{aligned}
$$

Then if $x$ is $C_{\lambda}^{*}$-summable to $L$,

$$
\begin{aligned}
0 \leq\left|\left(C_{\rho}^{*} x\right)_{n}-L\right| & \leq\left|\left(C_{\rho}^{*} x\right)_{n}-\left(C_{\lambda}^{*} x\right)_{n}\right|+\left|\left(C_{\lambda}^{*} x\right)_{n}-L\right| \\
& =o(1)+o(1)=o(1) .
\end{aligned}
$$


Similarly, if $x$ is $C_{\rho}^{*}$-summable to $L$, then

$$
\begin{aligned}
0 \leq\left|\left(C_{\lambda}^{*} x\right)_{n}-L\right| & \leq\left|\left(C_{\rho}^{*} x\right)_{n}-\left(C_{\lambda}^{*} x\right)_{n}\right|+\left|\left(C_{\rho}^{*} x\right)_{n}-L\right| \\
& =o(1)+o(1)=o(1) .
\end{aligned}
$$

Thus, $C_{\lambda}^{*}$ and $C_{\rho}^{*}$ are equivalent for bounded sequences.

ACKNOWLEDGMENT. The authors would like to thank the referee for several helpful suggestions that have improved the exposition of this work.

\section{REFERENCES}

[1] D. H. Armitage and I. J. Maddox, A new type of Cesàro mean, Analysis 9 (1989), no. 1-2, 195-206.

[2] A. Bikjalis and G. Jasjunas, Limit theorems in the metric of the spaces $L_{1}$ and $l_{1}$, Litovsk. Mat. Sb. 7 (1967), 195-218.

[3] N. H. Bingham, Tauberian theorems and the central limit theorem, Ann. Probab. 9 (1981), no. 2, 221-231.

[4] J. Boos, Classical and Modern Methods in Summability, Oxford Mathematical Monographs, Oxford University Press, Oxford, 2000.

[5] R. G. Cooke, Infinite Matrices and Sequence Spaces, Macmillan, London, 1950.

[6] J. A. Fridy, Submatrices of summability matrices, Int. J. Math. Math. Sci. 1 (1978), no. 4, 519-524.

[7] C. Goffman and G. M. Petersen, Submethods of regular matrix summability methods, Canad. J. Math. 8 (1956), 40-46.

[8] J. D. Hill, Some properties of summability. II, Bull. Amer. Math. Soc. 50 (1944), 227-230.

[9] M. K. Khan, Statistical methods in analysis. I. Some Tauberian theorems for absolute summability, Pakistan J. Statist. 7 (1991), no. 1, 21-32.

[10] W. Meyer-König, Untersuchungen über einige verwandte Limitierungsverfahren, Math. Z. 52 (1949), 257-304 (German).

[11] J. A. Osikiewicz, Equivalence results for Cesàro submethods, Analysis (Münich) 20 (2000), no. $1,35-43$.

[12] G. M. Petersen, Inclusion between limitation methods, Math. Z. 65 (1956), 494-496.

[13] B. Shawyer and B. Watson, Borel's Methods of Summability, Oxford Mathematical Monographs, The Clarendon Press, Oxford University Press, New York, 1994.

[14] W. F. Steele, Summability of infinite sequences by submatrix methods, Ph.D. Dissertation, University of Pittsburgh, Pennsylvania, 1961.

Jeffrey A. Osikiewicz: Department of Mathematical Sciences, Kent State University, Tuscarawas Campus, 330 University Dr. NE, New Philadelphia, OH 44663-9403, USA

E-mail address: josikiewicz@tusc.kent.edu

Mohammad K. Khan: Department of Mathematical Sciences, Kent State University, Kent, OH 44242-0001, USA

E-mail address: kazim@math . kent. edu 


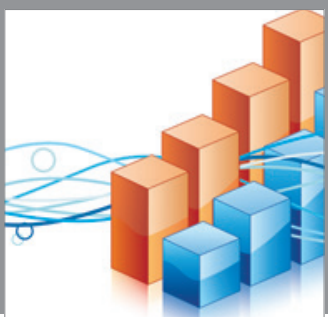

Advances in

Operations Research

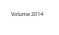

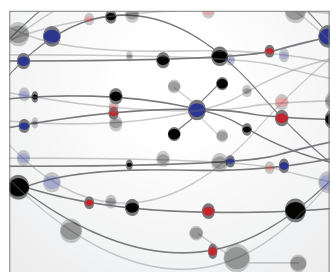

\section{The Scientific} World Journal
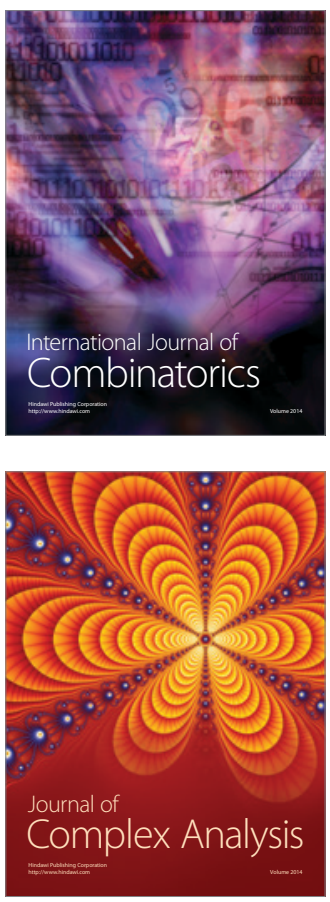

International Journal of

Mathematics and

Mathematical

Sciences
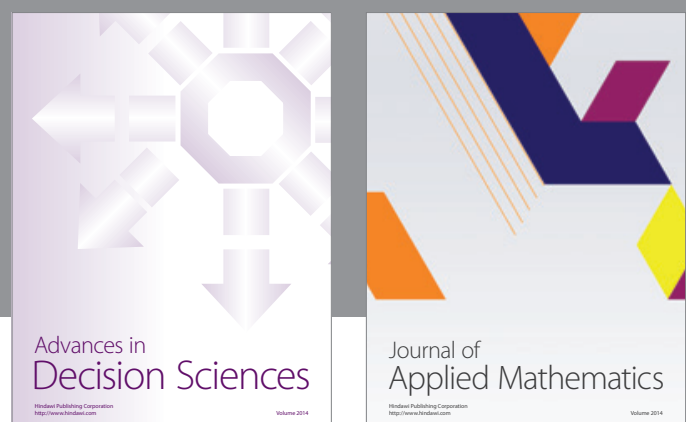

Journal of

Applied Mathematics
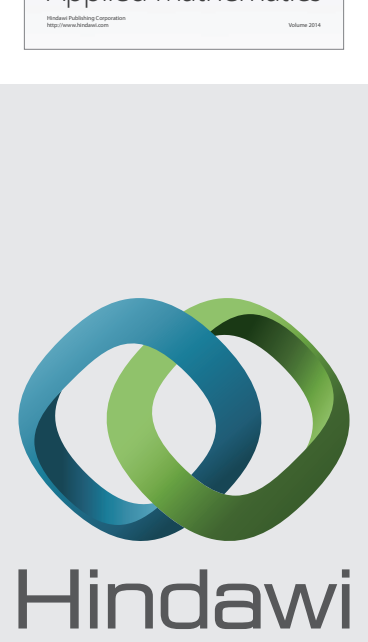

Submit your manuscripts at http://www.hindawi.com
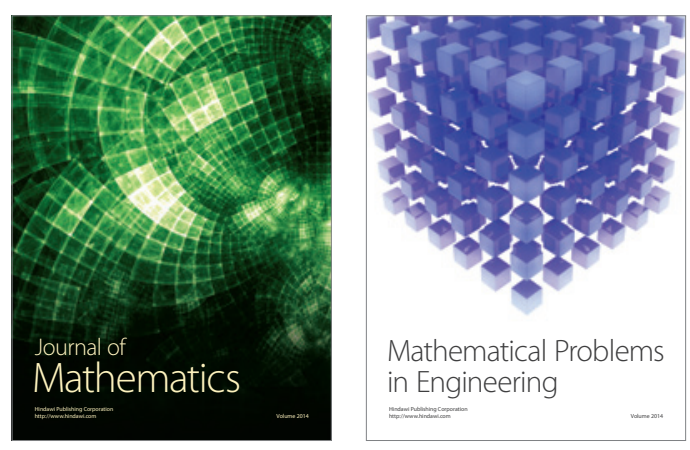

Mathematical Problems in Engineering
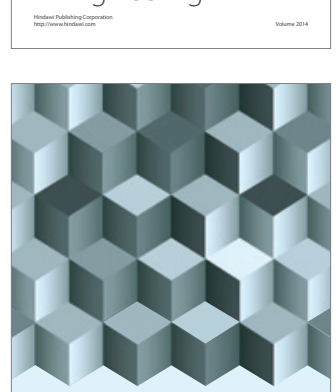

Journal of

Function Spaces
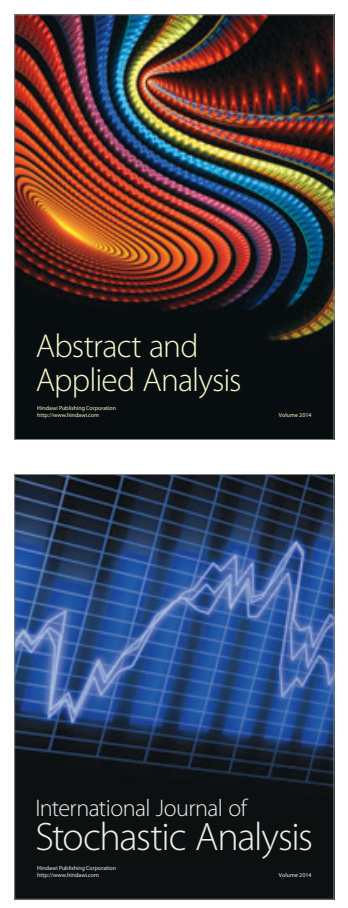

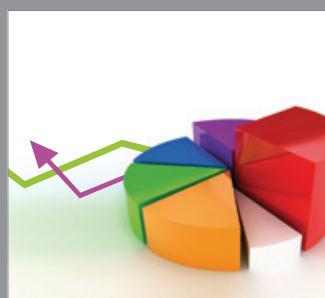

ournal of

Probability and Statistics

Promensencen
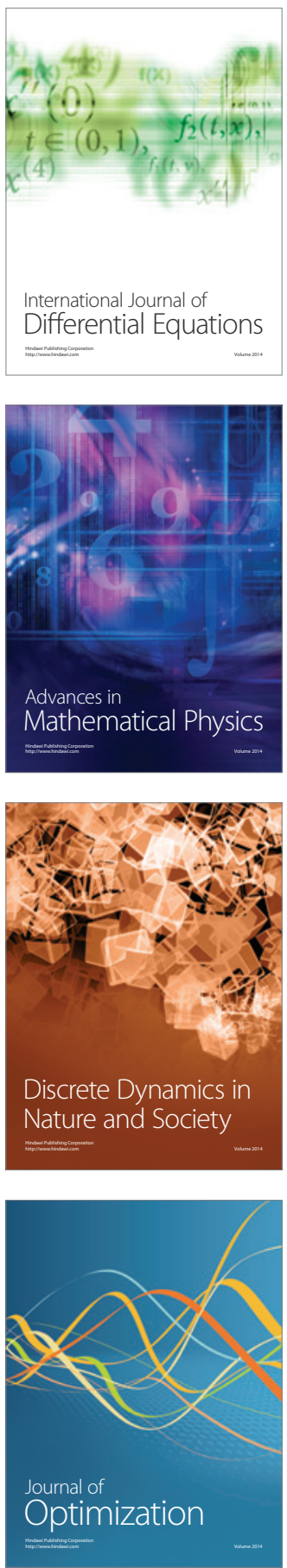